\title{
Avaliação bio econômica de frangos de corte alimentados com farelo integral de raízes de mandioca suplementado com carboidrases exógena
}

\author{
Bioeconomic evaluation of broilers fed meal of cassava roots supplemented with exogenous
}

carbohydrases

Evaluación bioeconómica de pollos de engorde alimentados con salvado entero de raíces de yuca suplementado con carbohidrasas exógenas

Wedja Edite de Oliveira Silva ORCID: https://orcid.org/0000-0001-7324-3108 Universidade Federal Rural de Pernambuco, Brasil E-mail: wedjaufrpe@hotmail.com

Marta Coelho Fernandes ORCID: https://orcid.org/0000-0002-6475-7227 Universidade Federal Rural de Pernambuco, Brasil E-mail: martaufrpe@hotmail.com

Camilla Letícia de Castro Silva

ORCID: https://orcid.org/0000-0002-5723-919X Universidade Federal Rural de Pernambuco, Brasil E-mail: kamyllacastro0506@hotmail.com

Antônio Daniel Lima do Nascimento

ORCID: https://orcid.org/0000-0003-1010-7752 Universidade Federal Rural de Pernambuco, Brasil

E-mail: danieldaniellima@outlook.com.br

Marco Aurélio Carneiro de Holanda

ORCID: https://orcid.org/0000-0002-9150-4487 Universidade Federal Rural de Pernambuco, Brasil E-mail: marco.holanda@ufrpe.br

Mônica Calixto Ribeiro de Holanda

ORCID: https://orcid.org/0000-0003-2197-4886 Universidade Federal Rural de Pernambuco, Brasil E-mail: monica.holanda@ufrpe.br

Leandro Ricardo Rodrigues de Lucena

ORCID: https://orcid.org/0000-0001-6985-7668 Universidade Federal Rural de Pernambuco, Brasil

E-mail: leandroricardo_est@yahoo.com.br

\begin{abstract}
Resumo
O experimento foi conduzido no aviário da Fazenda São João, localizada no distrito de Santa Rita, município de Serra Talhada-PE. Objetivou-se avaliar o desempenho de frangos de corte alimentados com dietas contendo diferentes níveis de inclusão de farelo integral de raízes de mandioca (FIRM), suplementadas com complexo multienzimático assim como a sua avaliação econômica. Foram utilizados 450 pintos de um dia de idade, machos da linhagem Cobb, distribuídas em 25 parcelas experimentais, em um delineamento inteiramente casualizado (DIC) com cinco tratamentos, compostos por quatro níveis de inclusão de 25, 50, 75 e 100\% de FIRM e uma dieta referência à base de milho e farelo de soja e cinco repetições por tratamento. O manejo adotado seguiu o preconizado no manual da linhagem Cobb. Foram mensuradas as variáveis de desempenho (ganho de peso médio diário, consumo de ração diário e conversão alimentar) e as variáveis econômicas receita bruta (RB), despesas (D), margem bruta (MB), e rentabilidade (REN). Os parâmetros de desempenho e de avaliação econômicas foram submetidos à análise de variância, sendo posteriormente realizadas análises de regressão e utilizado o teste Tukey com nível de significância a $5 \%$ de probabilidade $(\mathrm{P}<0,05)$. Foram observados melhora $(\mathrm{P}<0,05)$ no ganho de peso e conversão alimentar na fase de 36 a 42 dias e aumento $(\mathrm{P}<0,05)$ do ganho econômico. Concluiu-se que o FIRM suplementado com carboidrases exógenas pode ser incluído em dietas de frangos de corte industriais em até $100 \%$ produzindo melhora nos índices zootécnicos e aumento do ganho econômico em todo período experimental.
\end{abstract}

Palavras-chave: Amido resistente; Aves; Enzimas exógenas; Nutrição animal; Semiárido.

\footnotetext{
Abstract

The experiment was conducted in the aviary of Fazenda São João, located in the district of Santa Rita, municipality of Serra Talhada-PE. The objective was to evaluate the performance of broilers fed diets containing different levels of
} 
inclusion of manioc root bran (FIRM), supplemented with multi-enzyme complex. 450 day-old male chicks of the Cobb lineage were used, distributed in 25 experimental plots, in a completely randomized design (DIC) with five treatments, composed of four levels of inclusion of 25, 50, 75 and 100\% FIRM and a reference diet based on corn and soybean meal and five repetitions per treatment. The management adopted followed that recommended in the Cobb lineage manual. The performance variables (average daily weight gain, daily feed consumption and feed conversion) and the economic variables gross revenue (RB), expenses (D), gross margin (MB), and profitability (REN) were measured. The parameters of economic performance and evaluation were subjected to analysis of variance, later regression analyzes were performed and the Tukey test was used with a significance level at $5 \%$ probability $(\mathrm{P}<0.05)$. Improvements $(\mathrm{P}<0.05)$ in weight gain and feed conversion were observed in the 36 to 42 days phase and an increase $(\mathrm{P}<0.05)$ in economic gain. It was concluded that the FIRM supplemented with exogenous carbohydrates can be included in diets of industrial broilers by up to $100 \%$, producing improvement in zootechnical indexes and an increase in economic gain throughout the experimental period.

Keywords: Resistant starch; Poultry; Exogenous enzymes; Animal nutrition; Semiarid.

\section{Resumen}

El experimento se realizó en el aviario de Fazenda São João, ubicado en el distrito de Santa Rita, municipio de Serra Talhada-PE. El objetivo de este estudio fue evaluar el desempeño de pollos de engorde alimentados con dietas que contienen diferentes niveles de inclusión de harina integral de raíces de yuca (FIRM), suplementada con complejo multienzimático así como su evaluación económica. Se utilizaron pollos machos de 450 días de edad del linaje Cobb, distribuidos en 25 parcelas experimentales, en un diseño completamente aleatorizado (DIC) con cinco tratamientos, compuesto por cuatro niveles de inclusión de 25, 50, 75 y 100\% FIRM y una referencia. dieta a base de harina de maíz y soja y cinco repeticiones por tratamiento. La gestión adoptada siguió la recomendada en el manual de linaje Cobb. Se midieron las variables de desempeño (ganancia de peso diaria promedio, consumo diario de alimento y conversión alimenticia) y las variables económicas ingresos brutos (RB), gastos (D), margen bruto (MB) y rentabilidad (REN). Los parámetros de desempeño económico y evaluación fueron sometidos a análisis de varianza, posteriormente se realizaron análisis de regresión y se utilizó la prueba de Tukey con un nivel de significancia al 5\% de probabilidad $(\mathrm{P}<0.05)$. Se observó una mejora $(\mathrm{P}<0.05)$ en la ganancia de peso y conversión alimenticia en la fase de 36 a 42 días y un aumento $(\mathrm{P}<0.05)$ en la ganancia económica. Se concluyó que el FIRM suplementado con carbohidratos exógenos puede ser incluido en las dietas de pollos de engorde industriales hasta en un $100 \%$, produciendo una mejora en los índices zootécnicos y un aumento en la ganancia económica a lo largo del período experimental.

Palabras clave: Almidón resistente; Aves; Enzimas exógenas; Nutrición animal; Semiárido.

\section{Introdução}

A avicultura é um dos setores da produção animal mais desenvolvido nos últimos anos, principalmente no setor de produção de carne de frango (Henrique et al., 2017; Nogueira et al., 2019; Lucena et al., 2020).

Máxima eficiência alimentar e redução de custos em aves são pontos críticos a serem considerados em granjas comerciais porque um alimento devidamente balanceado e nutricionalmente completo reduzirá o estresse, minimizará deficiências, melhorará a competência imunológica e produzirá carcaças de qualidade com melhor desempenho e maior lucratividade (Pires et al., 2019; Lucena et al., 2020).

O custo da alimentação dos animais pode chegar a cerca de $70 \%$ do valor total gasto na produção e é afetado pelo preço dos grãos e ingredientes, como a soja, fazendo com que o setor produtivo utilize fontes alternativas de alimentos com custos mais baixos (Berwanger et al., 2017).

O preço do grão de milho tem oscilado consideravelmente, fazendo com que avicultores busquem alimentos alternativos para uso na alimentação avícola (Swain et al., 2006; Ashour et al., 2015; Niamat, 2017). Farinha de raiz de mandioca que tem sido usada como boa fonte alternativa de energia na dieta de aves e suínos (Diarra \& Devi, 2015; Kyawt et al., 2014; Zanu et al., 2017).

O uso da mandioca se destaca como ingrediente rico em carboidratos, fibra alimentar, amido, proteínas, lipídios e cinzas (Holanda et al., 2015), sendo capaz de compor dietas proporcionando ótimo ganho de peso e contribuindo para a redução do custo de produção de frangos de corte (Diarra \& Devi, 2015; Zanu et al., 2017). O farelo de mandioca pode ser utilizado em até $50 \%$ nas dietas de frango de corte em substituição ao milho sem comprometer o desempenho zootécnico das aves (Holanda et al., 2015; Enyenihi et al., 2009; Tesfaye et al., 2014) . 
Deste modo, objetivou-se avaliar o desempenho de frangos de corte industriais criados em clima semiárido alimentados com dietas contendo farelo integral de raízes de mandioca, suplementadas com pool de enzimas exógenas especificas para carboidratos.

\section{Metodologia}

A pesquisa foi realizada no aviário da Fazenda São João, localizada no distrito de Santa Rita, município de Serra Talhada-PE, na microregião do Sertão do Pajeú, mesorregião do Sertão de Pernambuco, sob a licença número 127/2019 do comitê de ética no uso de animais da Universidade Federal Rural de Pernambuco. A pesquisa teve o caráter de estudo quantitativo, caracterizada como pesquisa experimental, realizada por meio de técnicas de execução e análise de testes, avaliação numérica e processamento de dados por meio de técnicas estatísticas (Pereira et al., 2018).

Foram utilizados 450 pintos de corte da linhagem Cobb, machos, com um dia de idade, peso inicial de 42 gramas, vacinados no primeiro dia ainda no incubatório, contra Mareck, Newcastle, Gumboro e revacinados aos 14 dias contra Newcastle e Gumboro.

As aves foram alojadas em aviário construído em alvenaria, com telhas cerâmicas e piso de concreto, forrado com cama de material inerte (casca de arroz) à altura de $15 \mathrm{~cm}$, telado com tela de arrame galvanizado e cortinado para evitar correntes de ar e controlar a temperatura do ambiente. Durante os primeiros 14 dias de vida, foi utilizada uma lâmpada incandescente de 150 watts como fonte de calor para as aves. $\mathrm{O}$ aviário foi dividido em 25 parcelas experimentais, medindo cada uma dois metros quadrados, sendo que a densidade de criação foi de nove aves $/ \mathrm{m}^{2}$.

O delineamento experimental foi inteiramente casualizado com cinco tratamentos e cinco repetições, onde cada unidade experimental foi composta por 18 aves. Os tratamentos consistiram de uma ração testemunha à base de milho e farelo de soja, e quatro dietas testes contendo 25, 50, 75 e 100\% de inclusão de farelo integral de raízes de mandioca (FIRM) suplementados com enzimas endógenas, na quantidade de 500 gramas por tonelada de ração.

As raízes de mandioca foram adquiridas no município de Araripina-PE, processadas em moinho próprio para fabricação de farinhas de mesa e a massa desidratada ao sol por cinco dias até perder o máximo em umidade para obtenção do farelo seco, posteriormente uma amostra foi levada ao laboratório para analises bromatológicas e apresentou 88,56\% de matéria seca, 2,54\% de proteína bruta, 0,62 \% de lipídeos, 5,32\% de fibra bruta, 10,84\% de FDN e 3,96\% de FDA, 84,92\% de matéria orgânica, $3,52 \%$ de cinzas, $0,18 \%$ de cálcio e $0,09 \%$ de fósforo, a energia bruta foi determinada no calorímetro IKA 200 e foi obtido $4.123 \mathrm{kcal} / \mathrm{kg}$. Estes resultados de composição bromatológica foram utilizados para formulação das dietas experimentais juntamente com o resultado da energia metabolizável que foi determinada em um experimento de metabolismo realizado previamente com pintos determinando-se o valor de $2.986 \mathrm{Kcal} / \mathrm{kg}$.

O complexo multienzimático foi composto de galactosidase $35 \mathrm{U} / \mathrm{g}$, galactomananase $110 \mathrm{U} / \mathrm{g}$, xilanase $1.500 \mathrm{U} / \mathrm{g}, \beta$ glucanase $1.100 \mathrm{U} / \mathrm{g}$, e foi adicionado ao premix na proporção de 500 gramas por tonelada de ração, em um misturador tipo Y para mistura dos ingredientes de baixo nível de inclusão nas dietas.

A partir do primeiro dia de vida as aves receberam as dietas experimentais de acordo com os tratamentos, sendo a fase pré-inicial estabelecida do primeiro ao sétimo dia, a inicial dos oito aos 21 dias, a de crescimento dos 22 aos 35 dias e a de terminação dos 36 aos 42 dias, seguindo as Tabelas Brasileiras para Aves e Suínos - Composição de Alimentos e Exigências Nutricionais (Rostagno et al., 2017) e são apresentadas nas Tabelas 1, 2, 3, 4. 
Tabela 1. Composição das dietas experimentais, em função dos níveis de inclusão do farelo de mandioca no período de um a sete dias de idade.

\begin{tabular}{|c|c|c|c|c|c|}
\hline \multirow[b]{2}{*}{ Ingredientes } & \multicolumn{5}{|c|}{ Níveis de inclusão de mandioca (\%) } \\
\hline & 0 & 25 & 50 & 75 & 100 \\
\hline Milho & 46,543 & 34,907 & 23,271 & 11,635 & 0,000 \\
\hline Farelo de soja $45 \%$ & 46,129 & 47,743 & 49,360 & 50,977 & 52,594 \\
\hline Mandioca Raspa Integral & 0,000 & 8,888 & 17,777 & 26,665 & 35,554 \\
\hline Fosfato bicálcico & 1,930 & 2,201 & 2,524 & 2,846 & 3,169 \\
\hline Calcário Calcítico & 0,941 & 0,705 & 0,470 & 0,235 & 0,000 \\
\hline Óleo vegetal & 3,330 & 4,390 & 5,451 & 6,512 & 7,573 \\
\hline $\mathrm{NaCl}$ & 0,456 & 0,450 & 0,445 & 0,439 & 0,434 \\
\hline L-Lisina & 0,133 & 0,111 & 0,088 & 0,066 & 0,044 \\
\hline DL-Metionina & 0,328 & 0,348 & 0,368 & 0,388 & 0,408 \\
\hline L-treonina & 0,010 & 0,017 & 0,025 & 0,032 & 0,040 \\
\hline Complexo Multienzimatico & 0,000 & 0,050 & 0,050 & 0,050 & 0,050 \\
\hline Cloreto de Colina $60 \%$ & 0,100 & 0,100 & 0,100 & 0,100 & 0,100 \\
\hline Premix vitamínico/mineral & 0,100 & 0,100 & 0,100 & 0,100 & 0,100 \\
\hline \multicolumn{6}{|c|}{ Composição Calculada } \\
\hline $\mathrm{PB}(\%)$ & 25,310 & 25,310 & 25,310 & 25,310 & 25,310 \\
\hline $\mathrm{EM}(\mathrm{Kcal} / \mathrm{Kg})$ & 3.000 & 3.000 & 3.000 & 3.000 & 3.000 \\
\hline $\mathrm{Ca}(\%)$ & 1,011 & 1,011 & 1,011 & 1,011 & 1,011 \\
\hline P disponível (\%) & 0,482 & 0,535 & 0,589 & 0,642 & 0,696 \\
\hline Lisina digestível (\%) & 1,364 & 1,364 & 1,364 & 1,364 & 1,364 \\
\hline Metionina digestível (\%) & 0,669 & 0,680 & 0,692 & 0,703 & 0,715 \\
\hline Meti+Cistina digestível (\%) & 0,989 & 0,989 & 0,989 & 0,989 & 0,989 \\
\hline Treonina digestível (\%) & 0,773 & 0,773 & 0,773 & 0,773 & 0,773 \\
\hline Triptofano digestível (\%) & 0,296 & 0,304 & 0,312 & 0,320 & 0,328 \\
\hline Sódio (\%) & 0,227 & 0,227 & 0,227 & 0,227 & 0,227 \\
\hline Gordura (\%) & 5,642 & 5,781 & 5,921 & 6,060 & 6,200 \\
\hline
\end{tabular}

1Premix vitamínico/kg: Ácido Fólico 106,00 mg; Pantotênico $2.490 \mathrm{mg}$; Antifúngico $5.000 \mathrm{mg}$; Antioxidante $200 \mathrm{mg}$; Biotina 21mg; Coccidiostático $15.000 \mathrm{mg}$; Colina $118.750 \mathrm{mg}$; Vitamina K3 525,20 mg; Niacina 7.840 mg; Piridoxina 210 mg; Riboflavina1.660 mg, Tiamina 360 mg; Vitamina A 2.090.000 UI; Vitamina B12 123.750 mcg; Vitamina D3 525.000UI; Vitamina E 4.175 mg. Cu 2.000mg; I 190 mg; Mn 18.750 mg; Se 75 mg; Zn 12.500 mg.

Fonte: Autores. 
Tabela 2. Composição das dietas experimentais, em função dos níveis de inclusão do farelo de mandioca no período de oito a 21 dias de idade.

\begin{tabular}{|c|c|c|c|c|c|}
\hline \multirow[b]{2}{*}{ Ingredientes } & \multicolumn{5}{|c|}{ Níveis de inclusão de mandioca (\%) } \\
\hline & 0 & 25 & 50 & 75 & 100 \\
\hline Milho & 48,080 & 36,060 & 24,040 & 12,020 & 0,000 \\
\hline Farelo de soja $45 \%$ & 43,600 & 45,235 & 46,870 & 48,505 & 50,141 \\
\hline Mandioca Integral Raspa, kg & 0,000 & 9,355 & 18,710 & 28,065 & 37,420 \\
\hline Fosfato bicálcico & 1,679 & 1,661 & 1,694 & 1,726 & 1,760 \\
\hline Calcário Calcítico & 1,017 & 0,967 & 0,918 & 0,869 & 0,820 \\
\hline Óleo vegetal & 4,510 & 5,547 & 6,585 & 7,622 & 8,660 \\
\hline $\mathrm{NaCl}$ & 0,444 & 0,438 & 0,432 & 0,426 & 0,420 \\
\hline L-Lisina & 0,136 & 0,113 & 0,091 & 0,069 & 0,047 \\
\hline DL-Metionina & 0,327 & 0,348 & 0,369 & 0,390 & 0,412 \\
\hline L-treonina & 0,012 & 0,041 & 0,071 & 0,100 & 0,130 \\
\hline Complexo Multienzimático & 0,000 & 0,050 & 0,050 & 0,050 & 0,050 \\
\hline Cloreto de Colina $60 \%$ & 0,100 & 0,100 & 0,100 & 0,100 & 0,100 \\
\hline Premix vitamínico/mineral & 0,100 & 0,100 & 0,100 & 0,100 & 0,100 \\
\hline \multicolumn{6}{|c|}{ Composição Calculada } \\
\hline $\mathrm{PB}(\%)$ & 24,30 & 24,30 & 24,30 & 24,30 & 24,30 \\
\hline $\mathrm{EM}(\mathrm{Kcal} / \mathrm{Kg})$ & 3.100 & 3.100 & 3.100 & 3.100 & 3.100 \\
\hline $\mathrm{Ca}(\%)$ & 0,970 & 0,970 & 0,970 & 0,970 & 0,970 \\
\hline P disponível (\%) & 0,432 & 0,432 & 0,432 & 0,432 & 0,432 \\
\hline Lisina digestível (\%) & 1,306 & 1,306 & 1,306 & 1,306 & 1,306 \\
\hline Metionina digestível (\%) & 0,657 & 0,669 & 0,681 & 0,693 & 0,705 \\
\hline Meti+Cistina digestível (\%) & 0,966 & 0,966 & 0,966 & 0,966 & 0,966 \\
\hline Treonina digestível (\%) & 0,816 & 0,805 & 0,794 & 0,783 & 0,773 \\
\hline Triptofano digestível (\%) & 0,282 & 0,269 & 0,257 & 0,244 & 0,232 \\
\hline Sódio (\%) & 0,221 & 0,221 & 0,221 & 0,221 & 0,221 \\
\hline Gordura (\%) & 6,820 & 6,990 & 7,160 & 7,330 & 7,500 \\
\hline
\end{tabular}

1Premix vitamínico/kg: Ácido Fólico 106,00 mg; Pantotênico $2.490 \mathrm{mg}$; Antifúngico $5.000 \mathrm{mg}$; Antioxidante $200 \mathrm{mg}$; Biotina 21mg; Coccidiostático 15.000 mg; Colina 118.750 mg; Vitamina K3 525,20 mg; Niacina 7.840 mg; Piridoxina 210 mg; Riboflavina1.660 mg, Tiamina 360 mg; Vitamina A 2.090.000 UI; Vitamina B12 123.750 mcg; Vitamina D3 525.000UI; Vitamina E 4.175 mg. Cu 2.000mg; I 190 mg; Mn 18.750 mg; Se 75 mg; Zn 12.500 mg.

Fonte: Autores. 
Tabela 3. Composição das dietas experimentais, em função dos níveis de inclusão do farelo de mandioca no período de 22 a 35 dias de idade.

\begin{tabular}{|c|c|c|c|c|c|}
\hline \multirow[b]{2}{*}{ Ingredientes } & \multicolumn{5}{|c|}{ Níveis de inclusão de mandioca (\%) } \\
\hline & 0 & 25 & 50 & 75 & 100 \\
\hline Milho & 60,880 & 45,660 & 30,440 & 15,220 & 0,000 \\
\hline Farelo de soja $45 \%$ & 32,814 & 34,825 & 36,837 & 38,848 & 40,860 \\
\hline Mandioca Raspa Integral $30 \%$ & 0,000 & 12,560 & 25,135 & 37,702 & 50,270 \\
\hline Fosfato bicálcico & 1,420 & 1,407 & 1,445 & 1,482 & 1,520 \\
\hline Calcário Calcítico & 0,718 & 0,655 & 0,589 & 0,524 & 0,460 \\
\hline Óleo vegetal & 3,084 & 3,721 & 4,358 & 4,995 & 5,663 \\
\hline $\mathrm{NaCl}$ & 0,422 & 0,413 & 0,405 & 0,396 & 0,388 \\
\hline L-Lisina & 0,220 & 0,194 & 0,168 & 0,142 & 0,116 \\
\hline DL-Metionina & 0,272 & 0,299 & 0,327 & 0,364 & 0,394 \\
\hline L-treonina & 0,000 & 0,027 & 0,055 & 0,082 & 0,110 \\
\hline Complexo Multienzimatico & 0,00 & 0,050 & 0,050 & 0,050 & 0,050 \\
\hline Cloreto de Colina $60 \%$ & 0,100 & 0,100 & 0,100 & 0,100 & 0,100 \\
\hline Premix vitamínico/mineral & 0,100 & 0,100 & 0,100 & 0,100 & 0,100 \\
\hline \multicolumn{6}{|c|}{ Composição Calculada } \\
\hline $\mathrm{PB}(\%)$ & 20,58 & 20,58 & 20,58 & 20,58 & 20,58 \\
\hline $\mathrm{EM}(\mathrm{Kcal} / \mathrm{Kg})$ & 3.150 & 3.150 & 3.150 & 3.150 & 3.150 \\
\hline $\mathrm{Ca}(\%)$ & 0,758 & 0,758 & 0,758 & 0,758 & 0,758 \\
\hline P disponível (\%) & 0,374 & 0,374 & 0,374 & 0,374 & 0,374 \\
\hline Lisina digestível (\%) & 1,124 & 1,124 & 1,124 & 1,124 & 1,124 \\
\hline Metionina digestível (\%) & 0,557 & 0,572 & 0,588 & 0,603 & 0,619 \\
\hline Metio.+Cistina digestível (\%) & 0,832 & 0,832 & 0,832 & 0,832 & 0,832 \\
\hline Treonina digestível (\%) & 0,773 & 0,773 & 0,773 & 0,773 & 0,773 \\
\hline Triptofano digestível (\%) & 0,225 & 0,229 & 0,233 & 0,237 & 0,241 \\
\hline Sódio (\%) & 0,224 & 0,224 & 0,224 & 0,224 & 0,224 \\
\hline Gordura (\%) & 5,680 & 6,285 & 6,890 & 7,495 & 8,100 \\
\hline
\end{tabular}

1Premix vitamínico/kg: Ácido Fólico 106,00 mg; Pantotênico $2.490 \mathrm{mg}$; Antifúngico $5.000 \mathrm{mg}$; Antioxidante $200 \mathrm{mg}$; Biotina 21mg; Coccidiostático 15.000 mg; Colina 118.750 mg; Vitamina K3 525,20 mg; Niacina 7.840 mg; Piridoxina 210 mg; Riboflavina1.660 mg, Tiamina 360 mg; Vitamina A 2.090.000 UI; Vitamina B12 123.750 mcg; Vitamina D3 525.000UI; Vitamina E 4.175 mg. Cu 2.000mg; I 190 mg; Mn 18.750 mg; Se 75 mg; Zn 12.500 mg.

Fonte: Autores. 
Tabela 4. Composição das dietas experimentais, em função dos níveis de inclusão do farelo de mandioca no período de 36 a 42 dias de idade.

\begin{tabular}{|c|c|c|c|c|c|}
\hline \multirow[b]{2}{*}{ Ingredientes } & \multicolumn{5}{|c|}{ Níveis de inclusão de mandioca (\%) } \\
\hline & 0 & 25 & 50 & 75 & 100 \\
\hline Milho & 62,722 & 46,976 & 31,321 & 15,675 & 0,000 \\
\hline Farelo de soja $45 \%$ & 30,217 & 32,282 & 34,348 & 36,414 & 38,500 \\
\hline Mandioca raspa integral & 0,000 & 12,973 & 25,946 & 38,919 & 51,892 \\
\hline Fosfato bicálcico & 1,089 & 1,076 & 1,114 & 1,151 & 1,190 \\
\hline Calcário Calcítico & 0,701 & 0,634 & 0,568 & 0,501 & 0,435 \\
\hline Óleo vegetal & 4,218 & 4,856 & 5,494 & 6,132 & 6,770 \\
\hline $\mathrm{NaCl}$ & 0,407 & 0,398 & 0,390 & 0,381 & 0,373 \\
\hline L-Lisina & 0,226 & 0,199 & 0,173 & 0,146 & 0,120 \\
\hline DL-Metionina & 0,253 & 0,281 & 0,309 & 0,337 & 0,366 \\
\hline L-treonina & 0,064 & 0,075 & 0,087 & 0,098 & 0,110 \\
\hline Complexo multienzimatico & 0,000 & 0,050 & 0,050 & 0,050 & 0,050 \\
\hline Cloreto de colina $60 \%$ & 0,100 & 0,100 & 0,100 & 0,100 & 0,100 \\
\hline Premix vitamínico/mineral & 0,100 & 0,100 & 0,100 & 0,100 & 0,100 \\
\hline \multicolumn{6}{|c|}{ Composição Calculada } \\
\hline $\mathrm{PB}(\%)$ & 19,54 & 19,54 & 19,54 & 19,54 & 19,54 \\
\hline $\mathrm{EM}(\mathrm{Kcal} / \mathrm{Kg})$ & 3.250 & 3.250 & 3.250 & 3.250 & 3.250 \\
\hline $\mathrm{Ca}(\%)$ & 0,661 & 0,661 & 0,661 & 0,661 & 0,661 \\
\hline $\mathrm{P}$ disponível (\%) & 0,309 & 0,309 & 0,309 & 0,309 & 0,309 \\
\hline Lisina digestível (\%) & 1,067 & 1,067 & 1,067 & 1,067 & 1,067 \\
\hline Metionina digestível (\%) & 0,525 & 0,541 & 0,557 & 0,573 & 0,589 \\
\hline Met. + Cistina digestível (\%) & 0,790 & 0,790 & 0,790 & 0,790 & 0,790 \\
\hline Sódio (\%) & 0,201 & 0,201 & 0,201 & 0,201 & 0,201 \\
\hline Treonina digestível (\%) & 0,704 & 0,704 & 0,704 & 0,704 & 0,704 \\
\hline Gordura (\%) & 6,760 & 6,922 & 7,085 & 7,247 & 7,410 \\
\hline
\end{tabular}

1Premix vitamínico/kg: Ácido Fólico 106,00 mg; Pantotênico 2.490 mg; Antifúngico 5.000 mg; Antioxidante 200 mg; Biotina 21mg; Coccidiostático 15.000 mg; Colina $118.750 \mathrm{mg}$; Vitamina K3 525,20 mg; Niacina 7.840 mg; Piridoxina 210 $\mathrm{mg}$; Riboflavina1.660 mg, Tiamina $360 \mathrm{mg}$; Vitamina A $2.090 .000 \mathrm{UI}$; Vitamina B12 $123.750 \mathrm{mcg}$; Vitamina D3 525.000UI; Vitamina E 4.175 mg. Cu 2.000mg; I 190 mg; Mn 18.750 mg; Se 75 mg; Zn 12.500 mg.

Fonte: Autores.

Para a avaliação dos parâmetros de desempenho em função dos níveis de FIRM nas dietas, as aves e as rações foram pesadas a cada sete dias para mensuração do ganho de peso médio diário (GPMD, g/ave), consumo de ração diário (CRD, g/ave) e conversão alimentar (CA, g/g) e foram analisadas de acordo com a fase de produção e nos períodos de um a 21 dias, de 22 a 42 dias e no período de um a 42 dias de idade.

Ao final do experimento foi realizada a avaliação econômica da inclusão do farelo de raízes de mandioca com a apuração dos custos e das receitas relativas ao experimento de desempenho. Os preços das rações experimentais foram calculados com base nos preços dos ingredientes durante o período experimental e são apresentados na Tabela 5. 
Tabela 5. Preços das rações experimentais em reais na época do experimento de acordo com os níveis de inclusão do farelo integral de mandioca nas rações.

\begin{tabular}{lccccc}
\hline \multirow{2}{*}{ Rações } & \multicolumn{6}{c}{ Níveis de inclusão do Farelo Integral de Raízes de Mandioca } \\
\hline Pré inicial (0 a 7 dias) & $0 \%$ & $25 \%$ & $50 \%$ & $75 \%$ & $100 \%$ \\
Inicial (8 a 21 dias) & 1,531 & 1,478 & 1,365 & 1,352 & 1,321 \\
Crescimento (22 a 35 dias) & 1,533 & 1,479 & 1,424 & 1,370 & 1,316 \\
Final (36 a 42 dias) & 1,342 & 1,288 & 1,236 & 1,182 & 1,130 \\
Média & 1,364 & 1,304 & 1,124 & 1,184 & 1,125 \\
\hline
\end{tabular}

Fonte: Autores.

O cálculo da receita bruta foi realizado através da multiplicação do ganho de peso médio das aves no período pelo valor do quilo do frango vivo ao término do experimento que foi de $\mathrm{R} \$ 4,50$, representado pela equação:

$$
R=G P M * 4,50
$$

A despesa foi calculada através da multiplicação do consumo médio de ração no período pelo valor da ração de cada tratamento dividido por 0,7 , admitindo que a ração represente $70 \%$ do custo de produção do frango, e descrita pela equação:

$$
\mathrm{D}=\frac{\mathrm{CMR} * \text { valor da ração por tratamento }}{0,7}
$$

A margem bruta foi calculada subtraindo a despesa da receita, e definida por:

$$
M B=R-D
$$

A rentabilidade foi calculada pelo quociente entre a margem bruta pela despesa, e multiplicada por 100, definida pela equação abaixo:

$$
\mathrm{REN}=\left(\frac{\mathrm{MB}}{\mathrm{D}}\right) * 100
$$

As variáveis: ganho de peso (GP), consumo de ração (CR), conversão alimentar (CA), Receita (R), despesa (D), margem bruta (MB) e rentabilidade (REN) foram submetidas à análise de variância (ANOVA). As variáveis que apresentaram diferença significativa na ANOVA foram submetidas à análise de regressão, tomando como variável explicativa os diferentes níveis de inclusão de farelo de mandioca na dieta dos frangos de corte. Para todas as análises foi adota nível de significância de $5 \%(\mathrm{p}<0,05)$. Todas as análises foram realizadas utilizando o programa computacional R Project 2.13.1 for Windows ( $\mathrm{R}$ Core Team, 2019).

\section{Resultados e Discussão}

Nas fases pré-inicial (0 a 7 dias), inicial (8 a 21 dias) e crescimento (22 a 35 dias) não foram observadas diferenças estatísticas ( $>>0,05)$ entre os níveis de inclusão de farelo de mandioca nas dietas dos frangos de corte para as variáveis: ganho de peso, consumo de ração e conversão alimentar (Tabela 6). Na fase final da criação (36 a 42 dias) foram observadas diferenças estatísticas entre os tratamentos para as variáveis: ganho de peso (p-valor=0,001) e conversão alimentar (pvalor=0,032), porém não observou diferença para o consumo de ração (p-valor=0,194), Tabela 6. No período total de 
experimento ( 1 a 42 dias) não foram observadas diferença no ganho de peso (p-valor=0,55), consumo de ração (p-valor=0,476) e conversão alimentar ( $\mathrm{p}$-valor=0,451) quando comparadas as diferentes dietas (Tabela 6).

Tabela 6. Ganho de peso, consumo de ração e conversão alimentar de frangos de corte nas fases pré inicial, inicial, crescimento, final e período total em função dos níveis de inclusão do farelo integral de mandioca nas dietas.

\begin{tabular}{|c|c|c|c|c|c|c|}
\hline \multirow[b]{2}{*}{ Variáveis } & \multicolumn{6}{|c|}{ Níveis de inclusão de farelo de Raspa de Mandioca (\%) } \\
\hline & 0 & 25 & 50 & 75 & 100 & $\mathrm{p}$-valor \\
\hline \multicolumn{7}{|c|}{ Fase Pré Inicial (0 a 7 dias) } \\
\hline GP $(g)$ & $112,3 \pm 0,19$ & $113,8 \pm 0,97$ & $123,4 \pm 0,53$ & $120,0 \pm 0,51$ & $112,2 \pm 0,14$ & 0,289 \\
\hline $\mathrm{CR}(\mathrm{g})$ & $166,4 \pm 0,02$ & $166,2 \pm 0,03$ & $165,5 \pm 0,01$ & $165,5 \pm 0,02$ & $167,5 \pm 0,03$ & 0,312 \\
\hline $\mathrm{CA}(\mathrm{g} / \mathrm{g})$ & $1,48 \pm 0,14$ & $1,46 \pm 0,13$ & $1,34 \pm 0,05$ & $1,38 \pm 0,06$ & $1,49 \pm 0,20$ & 0,290 \\
\hline \multicolumn{7}{|c|}{ Fase Inicial ( 8 a 21 dias) } \\
\hline GP $(g)$ & $820,0 \pm 0,03$ & $831,7 \pm 0,04$ & $807,2 \pm 0,06$ & $798,0 \pm 0,05$ & $802,7 \pm 0,03$ & 0,791 \\
\hline $\mathrm{CR}(\mathrm{g})$ & $1083,6 \pm 0,01$ & $1072,0 \pm 0,04$ & $1057,9 \pm 0,07$ & $1051,7 \pm 0,08$ & $1056,7 \pm 0,10$ & 0,954 \\
\hline $\mathrm{CA}(\mathrm{g} / \mathrm{g})$ & $1,32 \pm 0,05$ & $1,29 \pm 0,12$ & $1,31 \pm 0,15$ & $1,32 \pm 0,18$ & $1,32 \pm 0,19$ & 0,997 \\
\hline \multicolumn{7}{|c|}{ Fase de Crescimento ( 22 a 35 dias) } \\
\hline $\mathrm{GP}(\mathrm{g})$ & $1464,3 \pm 0,05$ & $1455,3 \pm 0,10$ & $1472,4 \pm 0,08$ & $1443,4 \pm 0,12$ & $1449,6 \pm 0,04$ & 0,985 \\
\hline $\mathrm{CR}(\mathrm{g})$ & $2355,5 \pm 0,06$ & $2275,2 \pm 0,09$ & $2336,7 \pm 0,13$ & $2375,8 \pm 0,04$ & $2248,0 \pm 0,09$ & 0,170 \\
\hline $\mathrm{CA}(\mathrm{g} / \mathrm{g})$ & $1,61 \pm 0,06$ & $1,56 \pm 0,05$ & $1,58 \pm 0,03$ & $1,65 \pm 0,14$ & $1,55 \pm 0,04$ & 0,269 \\
\hline \multicolumn{7}{|c|}{ Fase Final (36 a 42 dias) } \\
\hline GP $(g)$ & $750,5 \pm 0,06 \mathrm{~d}$ & $837,9 \pm 0,06 b$ & $864,2 \pm 0,04 b$ & $933,7 \pm 0,03 a$ & $911,6 \pm 0,05 a$ & 0,001 \\
\hline $\mathrm{CR}(\mathrm{g})$ & $1455,6 \pm 0,10$ & $1511,6 \pm 0,09$ & $1541,3 \pm 0,08$ & $1583,6 \pm 0,07$ & $1537,8 \pm 0,07$ & 0,194 \\
\hline $\mathrm{CA}(\mathrm{g} / \mathrm{g})$ & $1,95 \pm 0,26 \mathrm{a}$ & $1,80 \pm 0,05 b$ & $1,78 \pm 0,10 b$ & $1,70 \pm 0,10 \mathrm{c}$ & $1,69 \pm 0,09 \mathrm{c}$ & 0,032 \\
\hline \multicolumn{7}{|c|}{ Período Total (1 a 42 dias) } \\
\hline GP $(g)$ & $3147,1 \pm 64$ & $3238,7 \pm 171$ & $3267,2 \pm 159$ & $3295,3 \pm 213$ & $3272,9 \pm 59$ & 0,550 \\
\hline $\mathrm{CR}(\mathrm{g})$ & $5061,2 \pm 118$ & $5025,0 \pm 199$ & $5101,4 \pm 175$ & $5176,5 \pm 103$ & $5010,0 \pm 168$ & 0,476 \\
\hline $\mathrm{CA}(\mathrm{g} / \mathrm{g})$ & $1,60 \pm 0,04$ & $1,55 \pm 0,03$ & $1,56 \pm 0,04$ & $1,57 \pm 0,12$ & $1,52 \pm 0,06$ & 0,451 \\
\hline
\end{tabular}

Fonte: Autores.

Os diferentes níveis de inclusão de farelo de mandioca nas dietas dos frangos de corte nas fases iniciais não afetam o comportamento zootécnico das aves, porém diverge dos observados por Souza et al., (2012), Geron et al., (2015) e Holanda et al., (2015) que observaram piora no desempenho zootécnico das aves durante a fase inicial com a inclusão de farelo integral de raízes de mandioca na dieta de aves tipo caipiras.

Contudo, corroboram com os observados por Fernandes et al., (2017) que observaram melhora no ganho de peso e na conversão alimentar das aves aos 21 dias de idade, com a melhora concomitante da digestibilidade da energia proveniente do carboidrato. A suplementação dietética com o complexo multienzimático composto apenas por carboidrases exógenas deste experimento, pode ter disponibilizado mais carboidratos solúveis para utilização pelas enzimas endógenas no lúmen intestinal implicando em maior utilização desses carboidratos para produção de energia melhorando o ganho em conversão de produtos que aumentam a deposição de massa corpórea. Segundo Slominski (2011) a adição de enzimas que degradam polissacarídeos pode liberar o amido encapsulado, por solubilização da parede celular, melhorando o acesso de enzimas digestivas e a disponibilidade dos nutrientes para o organismo animal (Slominski, 2011). 
Na fase final da criação (36 a 42 dias) observa-se um comportamento quadrático para o ganho de peso das aves em função dos diferentes níveis de inclusão de farelo de mandioca, tendo as aves maximizado seu ganho de peso em 920,18 gramas quando o nível ótimo de inclusão de farelo integral de mandioca nas dietas foi de 92\%, determinado pela equação GP $=750,9+3,68 x-0,02 x^{2}$ com poder de explicação de $91,2 \%$, Figura 1 .

Figura 1. Evolução do ganho de peso em função dos diferentes níveis de inclusão de farelo de mandioca.

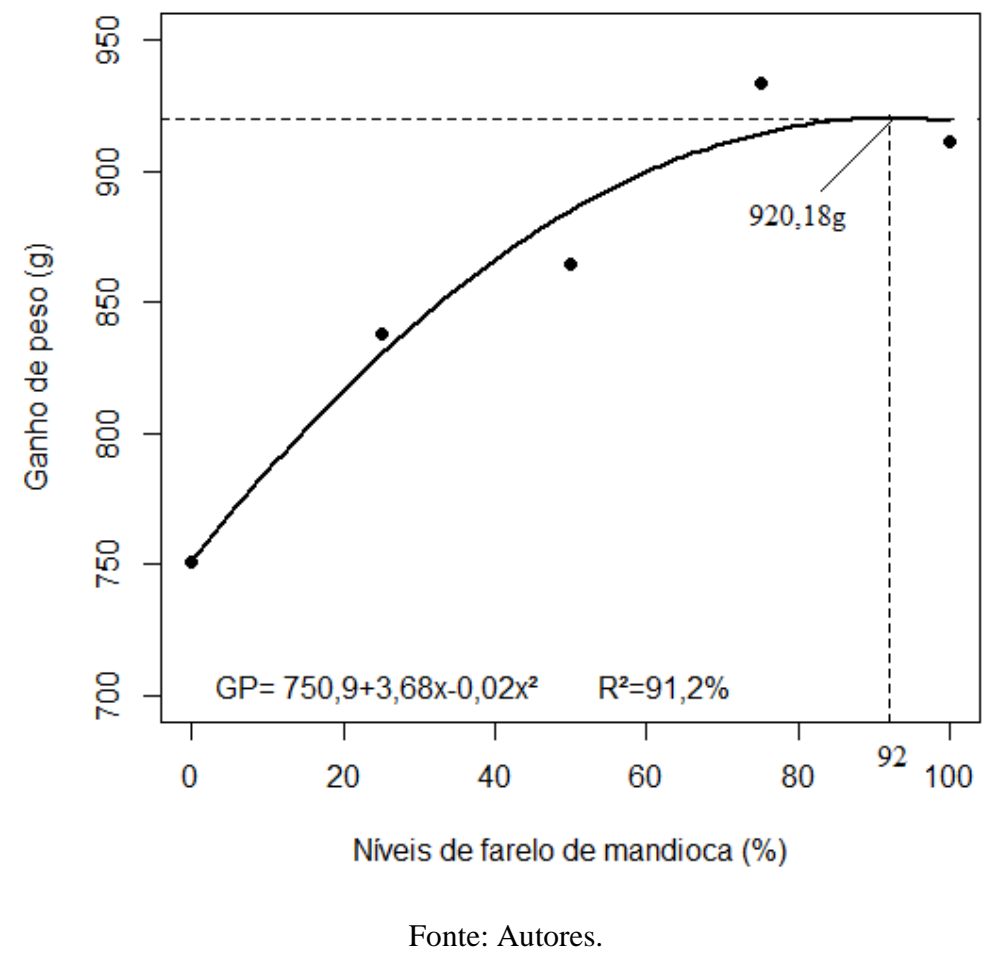

A conversão alimentar apresentou melhora linear em função do aumento dos níveis de inclusão de farelo de mandioca nas dietas das aves, e atingiu seu mínimo em 1,658 para um nível ótimo de 100\% de inclusão de farelo de mandioca, determinado pela equação $C A=1,908-0,0025 x$ com poder de explicação de 88,0 \%, Figura 2. 
Figura 2. Conversão alimentar das aves em função dos diferentes níveis de inclusão de farelo de mandioca.

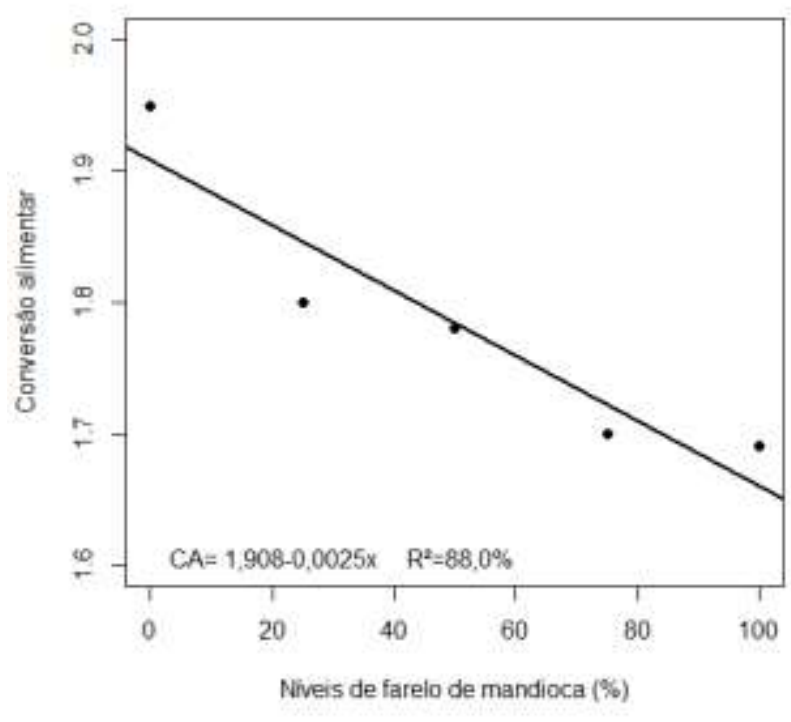

Fonte: Autores.

Os resultados apresentados no período total do experimento (1 a 42 dias) se mostraram mais eficazes que os relatados por: Carrijo et al., (2010), Souza et al., (2012), Geron et al., (2015), Holanda et al., (2015) e Fernandes et al., (2017) estes últimos, que avaliando a utilização de enzimas em dietas com milho classificado ou não, verificaram que não houve diferença entre os tratamentos, sugerindo que houve um efeito positivo da adição de enzimas sobre o consumo de ração, ganho de peso e conversão alimentar das aves. Tal fato pode provavelmente ter ocorrido, pois a suplementação do farelo integral de raízes de mandioca com enzimas exógenas tenha melhorado significativamente a digestibilidade dos carboidratos da mandioca, assim como dos demais ingredientes das dietas, produzindo um efeito melhorador sobre o consumo de ração, o ganho de peso e a conversão alimentar visto que houve efeito positivo da inclusão do farelo de mandioca nas dietas sobre o ganho de peso das aves.

A análise econômica da inclusão do farelo integral de raízes de mandioca nas dietas de frango de corte mostrou que não houve diferença estatística com a receita obtida com a venda dos frangos em todos os períodos avaliados (p-valor >0,05), porém para as variáveis: despesa, margem bruta e rentabilidade foram observadas diferenças estatísticas em função dos diferentes níveis de inclusão de farelo da mandioca nas dietas dos frangos de corte em todos os períodos avaliados (Tabela 7). 
Tabela 7. Médias das receitas, despesas, margem bruta e rentabilidade apuradas de acordo com os níveis de inclusão do farelo integral de raízes de mandioca nas dietas, nas fases de criação das aves.

\begin{tabular}{|c|c|c|c|c|c|c|}
\hline \multirow[b]{2}{*}{ Variáveis } & \multicolumn{6}{|c|}{ Níveis de inclusão de farelo de Raspa de Mandioca (\%) } \\
\hline & 0 & 25 & 50 & 75 & 100 & p-valor \\
\hline \multicolumn{7}{|c|}{ Fase Pré Inicial (1 a 7 dias) } \\
\hline Receita & $0,505 \pm 0,19$ & $0,512 \pm 0,97$ & $0,555 \pm 0,53$ & $0,540 \pm 0,51$ & $0,504 \pm 0,14$ & 0,289 \\
\hline Despesa & $0,361 \pm 0,02 \mathrm{a}$ & $0,348 \pm 0,03 b$ & $0,322 \pm 0,01 \mathrm{c}$ & $0,319 \pm 0,02 \mathrm{~cd}$ & $0,311 \pm 0,03 \mathrm{~d}$ & $<0,001$ \\
\hline Margem & $0,144 \pm 0,14 \mathrm{~d}$ & $0,163 \pm 0,13 c$ & $0,233 \pm 0,05 \mathrm{a}$ & $0,220 \pm 0,06 a b$ & $0,192 \pm 0,20 \mathrm{~b}$ & $<0,001$ \\
\hline Rentabilidade, $\%$ & $39,83 \pm 0,14 d$ & $46,74 \pm 0,14 \mathrm{c}$ & $72,34 \pm 0,14 \mathrm{a}$ & $69,17 \pm 0,14 \mathrm{a}$ & $61,83 \pm 0,14 b$ & $<0,001$ \\
\hline \multicolumn{7}{|c|}{ Fase Inicial ( 8 a 21 dias) } \\
\hline Receita & $3,69 \pm 0,03$ & $3,61 \pm 0,04$ & $3,63 \pm 0,06$ & $3,59 \pm 0,05$ & $3,61 \pm 0,03$ & 0,791 \\
\hline Despesa & $2,37 \pm 0,01 \mathrm{a}$ & $2,26 \pm 0,04 \mathrm{ab}$ & $2,15 \pm 0,07 b$ & $2,06 \pm 0,08 \mathrm{c}$ & $1,98 \pm 0,10 \mathrm{~d}$ & $<0,002$ \\
\hline Margem & $1,31 \pm 0,05 \mathrm{~d}$ & $1,47 \pm 0,12 \mathrm{c}$ & $1,48 \pm 0,15 c$ & $1,53 \pm 0,18 b$ & $1,62 \pm 0,19 a$ & 0,004 \\
\hline Rentabilidade, $\%$ & $55,49 \pm 0,05 c$ & $65,70 \pm 0,12 b$ & $69,44 \pm 0,15 b$ & $76,12 \pm 0,18 \mathrm{ab}$ & $83,70 \pm 0,19 a$ & 0,021 \\
\hline \multicolumn{7}{|c|}{ Fase de Crescimento (22 a 35 dias) } \\
\hline Receita & $6,59 \pm 0,26$ & $6,54 \pm 0,45$ & $6,62 \pm 0,37$ & $6,49 \pm 0,57$ & $6,52 \pm 0,19$ & 0,985 \\
\hline Despesa & $4,75 \pm 0,13 \mathrm{a}$ & $4,37 \pm 0,17 b$ & $4,26 \pm 0,25 b c$ & $4,09 \pm 0,07 \mathrm{c}$ & $3,65 \pm 0,15 d$ & $<0,001$ \\
\hline Margem & $1,83 \pm 0,25 \mathrm{c}$ & $2,18 \pm 0,28 b c$ & $2,37 \pm 0,16 b$ & $2,40 \pm 0,53 b$ & $2,87 \pm 0,13 \mathrm{a}$ & 0,269 \\
\hline Rentabilidade, $\%$ & $38,52 \pm 5,50 \mathrm{~d}$ & $49,77 \pm 4,70 \mathrm{c}$ & $55,73 \pm 3,20 b$ & $58,74 \pm 12,60 b$ & $78,92 \pm 5,10 \mathrm{a}$ & $<0,001$ \\
\hline \multicolumn{7}{|c|}{ Fase Final (36 a 42 dias) } \\
\hline Receita & $3,38 \pm 0,06 \mathrm{~d}$ & $3,77 \pm 0,06 c$ & $3,89 \pm 0,04 b c$ & $4,20 \pm 0,03 \mathrm{a}$ & $4,10 \pm 0,05 \mathrm{ab}$ & $<0,001$ \\
\hline Despesa & $2,79 \pm 0,10 \mathrm{a}$ & $2,78 \pm 0,09 \mathrm{a}$ & $2,72 \pm 0,08 \mathrm{a}$ & $2,67 \pm 0,07 \mathrm{ab}$ & $2,48 \pm 0,07 b$ & $<0,001$ \\
\hline Margem & $0,58 \pm 0,26 c$ & $0,98 \pm 0,05 b$ & $1,17 \pm 0,10 b$ & $1,53 \pm 0,10 \mathrm{a}$ & $1,62 \pm 0,09 a$ & $<0,001$ \\
\hline Rentabilidade, $\%$ & $21,62 \pm 42,0 \mathrm{c}$ & $35,48 \pm 3,82 b$ & $43,10 \pm 4,60 b$ & $57,40 \pm 5,20 \mathrm{a}$ & $65,30 \pm 4,80 a$ & $<0,001$ \\
\hline \multicolumn{7}{|c|}{ Período Total (1 a 42 dias) } \\
\hline Receita & $14,16 \pm 0,25$ & $14,57 \pm 0,77$ & $14,70 \pm 0,71$ & $14,83 \pm 0,95$ & $14,73 \pm 0,26$ & 0,550 \\
\hline Despesa & $10,38 \pm 0,24 a$ & $9,90 \pm 0,39 b$ & $9,36 \pm 0,32 \mathrm{c}$ & $9,30 \pm 0,18 \mathrm{c}$ & $8,62 \pm 0,29 d$ & $<0,001$ \\
\hline Margem & $3,77 \pm 0,29 \mathrm{e}$ & $4,68 \pm 0,41 d$ & $5,34 \pm 0,45 c$ & $5,52 \pm 1,00 \mathrm{~b}$ & $6,10 \pm 0,36 a$ & $<0,001$ \\
\hline Rentabilidade & $36,37 \pm 3,34 d$ & $47,20 \pm 2,89 \mathrm{c}$ & $56,97 \pm 3,75 b$ & $59,51 \pm 11,70 b$ & $71,00 \pm 6,18 \mathrm{a}$ & $<0,001$ \\
\hline
\end{tabular}

Fonte: Autores.

Na fase pré-inicial (01 a 07 dias) observou-se diminuição da despesa em $\mathrm{R} \$ 0,0005$ para cada ponto percentual de inclusão de farelo integral de raízes de mandioca nas dietas ( $\left.\hat{Y}=0,358-0,0005 x ; R^{2}=0,92\right)$ o que proporcionou uma melhora na margem bruta de aproximadamente $\mathrm{R} \$ 0,24$ quando o nível ótimo de inclusão de farelo integral de raízes de mandioca nas dietas foi de $66,4 \%$, determinado pela equação ( $\left.\hat{Y}=0,0073 x-0,000055 x^{2} ; R^{2}=0,89\right)$ indicando que os animais se mostraram intolerantes a níveis superiores ao determinado. Do mesmo modo, a rentabilidade observada aumentou, obtendo-se um rendimento de $75,08 \%$ quando a inclusão do farelo integral de raízes de mandioca nas dietas atingiu o nível ótimo de 68,5\% ( $\left.\hat{Y}=2,192 \mathrm{x}-0,016 \mathrm{x}^{2} ; \mathrm{R}^{2}=0,91\right)$.

$\mathrm{Na}$ fase inicial (08 a 21 dias) observou-se diminuição das despesas em $\mathrm{R} \$$ 0,0039 para cada ponto percentual de inclusão de farelo integral de raízes de mandioca nas dietas ( $\left.\hat{Y}=2,36-0,0039 x ; R^{2}=0,99\right)$, aumentando a margem bruta em $R \$$ 0,0675 para cada 25\% de inclusão do FIRM nas dietas, chegando ao valor de R\$ 1,62 quando foi incluído o nível 100\% de 
FIRM ( $\left.\hat{Y}=1,346+0,0027 x ; R^{2}=0,91\right)$, o que proporcionou um aumento na rentabilidade de $6,68 \%\left(\hat{Y}=56,722+0,267 x ; R^{2}=0,98\right)$ para cada $25 \%$ de inclusão do FIRM nas dietas, implicando num aumento da rentabilidade de $83,42 \%$ entre a ração referência e a dieta que continha o nível de inclusão $100 \%$.

$\mathrm{Na}$ fase de crescimento (22 a 35 dias) observou-se diminuição da despesa em $\mathrm{R} \$ 0,01$ para cada um ponto percentual de aumento na inclusão do FIRM nas dietas ( $\left.\hat{Y}=4,72-0,01 x ; R^{2}=0,95\right)$, e aumento na margem bruta de $R \$ 0,0092$ para cada ponto percentual de inclusão do FIRM na dieta, implicando em R \$ 0,92 de ganho a mais quando a inclusão for de $100 \%$ de FIRM ( $\left.\hat{Y}=1,87+0,0092 x ; R^{2}=0,93\right)$, o que reflete sobre a taxa de rentabilidade de aproximadamente $0,359 \%$ a mais para cada um ponto percentual de inclusão do FIRM na dieta ( $\hat{Y}=38,382+0,3591 x ; R^{2}=0,92$ ), implicando em ganho de 93,5\% quando comparamos os ganhos entre o tratamento da dieta referência e o ganho da dieta com 100\% de inclusão.

$\mathrm{Na}$ fase final (36 a 42 dias) observou-se diminuição das despesas em $\mathrm{R} \$$ 0,003 para cada um ponto percentual de aumento na inclusão de FIRM nas dietas ( $\left.\hat{Y}=2,834-0,003 x ; R^{2}=0,84\right)$ e aumento na margem bruta de $R \$ 0,0105$, implicando em R\$ 1,05 de ganho a mais quando o nível de inclusão for de $100 \%$ ( $\left.\hat{Y}=0,65+0,0105 x ; R^{2}=0,97\right)$, o que repercute positivamente sobre a taxa de rentabilidade que chegou a 66,4\% quando o nível de FIRM nas dietas é de $100 \%$ $\left(\hat{Y}=22,724+0,437 x ; R^{2}=0,99\right)$, implicando em um ganho de $192,3 \%$ quando comparamos os ganhos entre o tratamento da dieta referência e o ganho da dieta com $100 \%$ de inclusão do FIRM.

Para o período total de criação (01 a 42 dias) as despesas diminuíram em $\mathrm{R} \$$ 0,0165 para cada ponto percentual de inclusão de FIRM ( $\left.\hat{Y}=10,336-0,0165 x ; R^{2}=0,96\right)$, este efeito repercutiu positivamente sobre a margem bruta que aumentou em $\mathrm{R} \$ 2,2$ para o nível de $100 \%$ de inclusão $\left(\hat{\mathrm{Y}}=3,982+0,022 \mathrm{x} ; \mathrm{R}^{2}=0,95\right)$. A taxa de rentabilidade aumentou em $0,326 \%$ para cada aumento de $1 \%$ de inclusão de FIRM ( $\hat{Y}=37,896+0,326 x ; R^{2}=0,97$ ), implicando em aumento da rentabilidade de $86,0 \%$ do tratamento referência para o tratamento com $100 \%$ de inclusão de FIRM.

A medida que os níveis de FIRM aumentaram nas dietas do nível 0 para o nível 100\%, observou-se aumento na inclusão do farelo de soja e dos aminoácidos industriais nas dietas (Tabelas 1, 2, 3 e 4) isto provocado pela necessidade do balanceamento dos teores de proteína e consequentemente da disponibilidade de aminoácidos digestíveis, em todas as fases de criação. No entanto, a diferença entre os preços do milho em grãos e do FIRM desidratado e processado foi tão grande que a inclusão do FIRM nas dietas provocou diminuição nos preços das rações experimentais do nível 0 para o nível $100 \%$ em todas as fases avaliadas conforme observado na Tabela 5.

Estes resultados divergem dos observados por Souza et al. (2012) que observaram aumento no custo das dietas que continham bagaço de mandioca em função do aumento da inclusão dos aminoácidos industriais que aumentaram consideravelmente o preço das dietas experimentais o que levou os autores a apuração de resultados econômicos negativos na fase inicial de criação, e também são superiores aos observados por Costa et al. (2009) que avaliando o ganho econômico produzido pela inclusão de mandioca em dietas de poedeiras comerciais observaram ganhos com a inclusão de até $15 \%$ de mandioca em detrimento do uso do milho nas dietas.

Lucena et al. (2020) verificaram que a substituição farelo de mandioca na suplementação alimentar de frangos de corte, além de promover melhor desempenho zootécnico das aves, diminui o custo da produção em $\mathrm{R} \$ 0,06$ por quilo da ração. Souza et al. (2012) trabalhando com bagaço de mandioca em dietas para frangos de corte observaram prejuízo financeiro quando o nível de inclusão do bagaço de mandioca nas dietas foi superior a 20\%. Melo et al. (2017) avaliando a utilização da farinha de cará na alimentação de poedeiras comerciais concluíram que o nível de inclusão de 5\% produz o melhor desempenho econômico, indicando que níveis acima de 15\% de inclusão nas dietas provocam perdas econômicas significativas. Estes resultados são divergentes dos encontrados nessa pesquisa, onde o aumento da inclusão de farelo de mandioca proporcionou redução dos custos além de aumentar a rentabilidade. 
Os ganhos econômicos observados neste experimento mostram-se extremamente importantes principalmente para produtores situados em regiões onde haja produção de mandioca em quantidades que possa atender a demanda da produção avícola, pois na época da realização deste experimento o preço do quilo do milho foi de $\mathrm{R} \$ 1,00$ e o preço do quilo da raiz de mandioca desidratada e processada de $\mathrm{R} \$ 0,14$, o que proporcionou uma redução nos custos de produção significativa. Costa et al. (2009) e Rufino et al. (2015) relatam que antes da tomada de decisões quanto a utilização de um alimento alternativo em escala industrial de produção, deve-se levar em consideração os resultados da análise de rentabilidade e lucratividade deste nas rações, e se possível, constatar a uma relação positiva entre a análise nutricional e a análise produtiva, obtendo reduções significativas de custo e ganhos potenciais.

\section{Conclusão}

O farelo integral de raízes de mandioca suplementado por carboidrases exógenas pode ser utilizado na dieta de frangos de corte em todas as fases da criação em detrimento do uso do milho, sem prejuízo ao desempenho zootécnico e promovendo ganhos econômicos pela redução nos custos de produção da ração.

Trabalhos futuros devem ser realizados com outras fontes de alimentos alternativos em substituição do milho em dietas de poedeiras visando minimizar os custos de produção da ração.

\section{Referências}

Ademeri, F. A., Adenowo, T. K. \& Oguntunji, A. O. (2012). Efeito da Farinha de Mandioca nas Características de Desempenho e Qualidade dos Ovos de Camadas. Journal of Agricultural Science, 4, 195-200.

Ashour, E. A., Reda, F. M. \& Alagawany, M. (2015). Effect of graded replacement of corn by broken rice in growing Japanese quail diets on growth performance, carcass traits and economics. Asian Journal Animal Sciences, 9(6), 404-411.

Berwanger, E., Nunes, R. V., Oliveira, T. M. M., Bayerle, D. F. \& Bruno, L. D. G. (2017). Performance and carcass yield of broilers fed increasing levels of sunflower cake. Caatinga, 30(1), 201-212.

Carrijo, A. S., Fascina, V. B., Souza, K. M. R., Ribeiro, S. S., Allaman, I. B., Garcia, A. M. L. \& Higa, J. A. (2010). Níveis de farelo da raiz integral de mandioca em dietas para fêmeas de frangos caipiras. Revista Brasileira de Saúde e Produção Animal, 11(1), $131-139$.

Costa, F. G. P., Goulart, C. C., Costa, J. S., Souza, C. J., Barros, L. R. \& Silva, J. H. V. (2009). Desempenho, qualidade de ovos e análise econômica da produção de poedeiras semipesadas alimentadas com diferentes níveis de raspa de mandioca. Acta Scientiarum. Animal Science, 31(1),13-18.

Diarra, S. S. \& Devi A. (2015). Feeding value of some cassava by-products meal for poultry: a review. Pakistan Journal of Nutrition, 14(10), 735-741.

Enyenihi, G. E., Udedibie, A. B. I., Akpan, M. J., Obasi, O. L. \& Solomon, I. P. (2009). Effects of 5 h wetting of sun-dried cassava tuber meal on the hydrocyanide contente and dietary value of the meal for laying hens. Asian Journal of Animal and Veterinary Advances, $4,326-331$.

Fernandes, J. I. M., Contini, J. P., Prokoski, K., Gottardo, E. T., Cristo, A. B. \& Perini, R. (2017). Desempenho produtivo de frangos de corte e utilização de energia e nutrientes de dietas iniciais com milho classificado ou não e suplementadas com complexo enzimático. Arquivo Brasileiro de Medicina Veterinária e Zootecnia, 69(1), 181-190.

Geron, L. J. V., Costa, F. G., Moraes, K. J. S., Oliveira, E. M., Gomes, R. D., Pereira, S. R., Silva, A. P., Cruz, C. \& Pelícia, K. (2015). Consumo, desempenho e rendimento de carcaça de frangos de corte alimentados com rações contendo raspa de mandioca residual desidratada. Boletim Indústria Animal, 72(4), 304310 .

Henrique, C. S., Oliveira, A. F. G., Ferreira, T. S., Silva, E. S., Mello, B. F. F. R., Andrade, A. F., Martins, V. S. F., Paula, F. O., Garcia, E. R. M. \& Bruno, L. D. G. (2017). Effect of stocking density on performance, carcass yield, productivity, and bone development in broiler chickens Cobb 500. Semina: Ciências Agrárias, 38(4): 2705-2718.

Holanda, M. A. C., Holanda, M. C. R., Vigoderes, R. B., Dutra Junior, W. M. \& Albino, L. F. T. (2015). Desempenho de frangos caipiras alimentados com farelo integral de mandioca. Revista Brasileira de Saúde e Produção Animal, 16(1), 106-117.

Kyawt, Y. Y., Toyama, H., Htwe, W. M., Thaikua, S., Imura, Y. \& Kawamoto Y. (2014). Effects of Cassava Substitute for Maize Based Diets on Performance Characteristics and Egg Quality of Laying Hens. International Journal of Poultry Science, 13(9), 518-524.

Lucena, L. R. R., Holanda, M. A. C. \& Holanda, M. C. R. (2020). Growth curve of broiler chicken submitted the cassava meal diet. Cuban Journal of Agricultural Science, 54(3), 375-386.

Melo, R. D., Cruz, F. G. G., Feijó, J. C., Rufino, J. P. F., Melo, L. D. \& Costa, A. P. G. C. (2017). Viabilidade econômica da farinha de cará na alimentação de poedeiras comerciais leves. Revista Brasileira de Saúde e Produção Animal, 18(2), 221-230. 
Research, Society and Development, v. 10, n. 7, e7810716413, 2021

(CC BY 4.0) | ISSN 2525-3409 | DOI: http://dx.doi.org/10.33448/rsd-v10i7.16413

Niamat, M. E. A. (2017). Yellow corn replaced by distillers dried grains with solubles (ddgs) of dietary Japanese quail. Egyptian Poultry Science, 37(2), 451460 .

Nogueira, B. R. P., Reis, M. P., Carvalho, A. C., Mendoza, E. A. C., Oliveira, B. L., Silva, V. A. \& Bertechini, A. G. (2019). Performance, growth curves and carcass yield of four strains of broiler chicken. Brazilian Journal of Poultry Science, 21(4): 1-8.

Pereira A.S. et al. (2018). Metodologia da pesquisa científica. UFSM.

Pires, G. A., Cordeiro, M. B., Freitas, H. J., Rodrigues, S. F. C. \& Nascimento, A. M. (2019). Desempenho zootécnico e rendimento de carcaça de linhagens de frangos de corte criadas sob condições ambientais da Amazônia ocidental. Enciclopédia Biosfera, 16(29), 633-645.

R Core Team. (2019). R. A language and environment for statistical computing. Vienna, AU: Foudation for Statistical Computing. Retrieved from https://www.R-project.org/

Rostagno, H. S., Albino, L. F. T., Hannas, M. I., Donzele, J. L., Sakomura, N. K., Perazzo, F. G., Saraiva, A., Abreu, M. L. T., Rodrigues, P. B., Oliveira, R. F., Barreto, S. L. T. \& Brito, C. O. (2017). Tabelas Brasileiras para aves e suínos: composição de alimentos e exigências nutricionais. $4^{\mathrm{a}}$ Ed.

Rufino, J. P. F., Cruz, F. G. G., Miller, W. P. M., Melo, R. D., Feijó, J. C. \& Chagas, E. O. (2015). Análise econômica da inclusão de farinha do resíduo de tucumã (Astrocaryum vulgare Mart) na alimentação de poedeiras comerciais. Revista Brasileira de Saúde e Produção Animal, 16(1), 1-9.

Slominski, B. A. (2011). Recent advances in research on enzymes for poultry diets. Poultry Science, 90(9), 2013-2023.

Souza, J. P. L., Rodrigues, K. F., Albino, L. F. T., Santos Neta, E. R., Vaz, R. G. M. V., Parente, I. P., Silva, G. F. \& Amorim, A. F. (2012). Bagaço de mandioca em dietas de frangos de corte. Revista Brasileira de Saúde e Produção Animal, 13(4), 1044-1053.

Swain, B. K., Sundaram, R. N. S., Chakurkar, E. B. \& Barduddhe, S. B. (2006). Feeding value of broken rice for Japanese quail layers. Indian Journal of Animal Nutrition, 23(3), 193-195.

Tesfaye, E. B., Animut, G. M., Urge, M. L. \& Dessie, T. A. (2014). Cassava root chip and Moringa oleifera leaf meal as alternative feed ingredients in the layer ration. Journal of Applied Poultry Reserch, 23(4), 614-624.

Zanu, H. K., Azameti, M. K. \& Asare, D. (2017). Effects of dietary inclusion of cassava root flour in broiler diets on growth performance, carcass characteristic and haematological parameters. International Journal of Livestock Production, 8(3), 28-32. 